Article

\title{
Classification of Warped Product Submanifolds in Kenmotsu Space Forms Admitting Gradient Ricci Solitons
}

\author{
Ali H. Alkhaldi and Akram Ali *(D) \\ Department of Mathematics, College of Science, King Khalid University, 9004 Abha, Saudi Arabia; \\ ahalkhaldi@kku.edu.sa \\ * Correspondence: akramali133@gmail.com or akali@kku.edu.sa; Tel.: +966-554146618
}

Received: 2 January 2019; Accepted: 18 January 2019; Published: 22 January 2019

\begin{abstract}
The purpose of this article is to obtain geometric conditions in terms of gradient Ricci curvature, both necessary and sufficient, for a warped product semi-slant in a Kenmotsu space form, to be either CR-warped product or simply a Riemannian product manifold when a basic inequality become equality. The next purpose of this paper to find the necessary condition admitting gradient Ricci soliton, that the warped product semi-slant submanifold of Kenmotsu space form, is an Einstein warped product. We also discuss some obstructions to these constructions in more detail.
\end{abstract}

Keywords: warped products; Kenmotsu space forms; Euler-Lagrange equation; Ricci curvature; gradient Ricci soliton; gradient Ricci soliton warped product

\section{Introduction and Motivations of the Main Results}

As a generalization of Riemannian product manifolds, the warped product manifolds are defined as follows:

Definition 1. Let $\left(N_{1}, g_{1}\right)$ and $\left(N_{2}, g_{2}\right)$ be two Riemannian manifolds and $f: N_{1} \rightarrow(0, \infty)$, a positive differentiable function on $N_{1}$ which is known as the warping function. Then consider the product manifold $N_{1} \times N_{2}$ with its canonical projections $\gamma_{1}: N_{1} \times N_{2} \rightarrow N_{1}, \gamma_{2}: N_{1} \times N_{2} \rightarrow N_{2}$ and the projection maps given by $\gamma_{1}(t, s)=t$ and $\gamma_{2}(t, s)=s$ for every $l=(t, s) \in N_{1} \times N_{2}$. The warped product $M=N_{1} \times{ }_{f} N_{2}$ is the product manifold $N_{1} \times N_{2}$ equipped with the Riemannian structure such that $\|X\|^{2}=\left\|\gamma_{1} *(X)\right\|^{2}+$ $f^{2}\left(\gamma_{1}(t)\right)\left\|\pi_{2} *(X)\right\|^{2}$ with the Riemannian metric on $M g=g_{1}+f^{2} g_{2}$, for any tangent vector $X \in \mathfrak{X}\left(T_{t} M\right)$.

The following definitions can be seen as a conclusion of the warped product manifolds:

Definition 2. If the warping function $f$ is constant, then the warped product manifold $M=N_{1} \times_{f} N_{2}$ is said to be trivial or in other words is called simply a Riemannian product.

By applying a new method under the assumption of the Gauss equation instead of the Codazzi equation, it was proved in [1], every warped product semi-slant submanifold $M^{n}=N_{T}^{n_{1}} \times_{f} N_{\theta}^{n_{2}}$ isometrically immersed of a Kenmotsu space form $\widetilde{M}^{2 m+1}(c)$ with constant holomorphic sectional curvature $c$ satisfied the following optimal inequality

$$
\|h\|^{2} \geq 2 n_{2}\left(\|\nabla \ln f\|^{2}+\frac{c-3}{4} n_{1}-\frac{c+1}{4}-\Delta(\ln f)\right) .
$$


Moreover, the equality sign holds in (1) if and only if $N_{T}^{n_{1}}$ is totally geodesic and $N_{\theta}^{n_{1}}$ is totally umbilical submanifolds of $\tilde{M}^{2 m+1}(c)$, respectively and $M^{n}$ is minimal submanifold in $\widehat{M}^{2 m+1}(c)$. By considering the equality case in the statement of the inequality (1), we proved some results on the triviality of the warped product semi-slant submanifolds of Kenmotsu space forms.

Theorem 1. [1] Assume that $\chi: M^{n}=N_{T}^{n_{1}} \times_{f} N_{\theta}^{n_{2}} \longrightarrow \widetilde{M}^{2 m+1}(c)$ is an isometric immersion of a compact oriented proper warped product semi-slant submanifold $N_{T}^{n_{1}} \times_{f} N_{\theta}^{n_{2}}$ into a Kenmotsu space form $\tilde{M}^{2 m+1}(c)$. Then $M^{n}$ is simply a Riemannian product if and only if

$$
\sum_{i=1}^{n_{1}} \sum_{j=1}^{n_{2}}\left\|h_{\mu}\left(e_{i}, e_{j}\right)\right\|^{2}=n_{2}\left(\frac{c-3}{4} n_{1}-\frac{c+1}{4}+2 \cot ^{2} \theta+1\right)
$$

where $\theta$ is a slant angle defined on $T M$ and $h_{\mu}$ is the second fundamental form of the component in $\Gamma(\mu)$.

The above classification comes from the motivation of case study [2-8]. Moreover, such results are also presented in [1,4,9] of different ambient space forming and some of them as corollaries. We continue our study of ends of connected and compact manifolds, with a focus on the case that the derived inequality satisfys the equality condition. That observation leads to the further analysis of the equality conditions with compact boundary. We obtain a complete classification of compact warped product which admits steady and shrinking gradient Ricci Soliton, and gradient Ricci curvature structure, thereby expanding our previous work.

Furthermore, very much attention has been paid to hypothesizing the Einstein metric and its generalization from recent years, for instance, Ricci soliton and quasi-Einstein metrics. Einstein manifolds have been discussed extensively due to their connection with scalar curvature, positive mass theorem, and general relativity. In this direction, Ricci solitons are fixed points of Ricci flow as a dynamical system on the space of Riemannian metric modulo diffeomorphism and scaling. In the context of the fundamental equation, they are the natural generalization of Einstein manifolds. Moreover, Ricci soliton is important to understand singularities of Ricci flow as they appear as the self-similar solution of the Ricci flow $\frac{\partial}{\partial} g(t)=-2$ Ric $(t)$. The uniqueness of Ricci flow on closed manifolds was originally proved by Hamilton in [10] and for more detail (see [11,12]). If there exists a smooth vector field $X$ such that the Ricci tensor satisfies the following condition.

$$
\operatorname{Ric}+\frac{1}{2} \mathcal{L}_{X} g=\lambda
$$

for any constant $\lambda$, where $\mathcal{L}_{X}$ is the Lie derivative, then Riemannian metric $g$ on a complete Riemannian manifold $M^{n}$ is called a Ricci soliton. A Ricci soliton is called expanding, steady and shrinking if $\lambda<0, \lambda=0$, and $\lambda>0$, respectively. If we choose $X=\nabla f$ for a smooth function $f$ defined on $M^{n}$, then $M^{n}$ is called gradient Ricci soliton with $f$ being the potential function. In this case, the fundamental equation can be rewritten as:

$$
\operatorname{Ric}+\nabla^{2} f=\lambda g
$$

where $\nabla^{2} f$ denotes the Hessian of $f$. Due to the relation of the Laplacian $\Delta$ and the gradient $\nabla^{2}$ such that $\Delta=\nabla^{2}$. Thus, Equation (4) can be modified as;

$$
\operatorname{Ric}=\lambda g+\operatorname{Hess}(f) \text {. }
$$

It is also called the fundamental equation of Ricci tensor in terms of Hessian tensor. For the gradient, Ricci soliton can be classified as: 
Remark 1. If the potential function $f$ is a constant on gradient Ricci soliton, then $(M, g, \nabla f, \lambda)$ is an Einstein manifold.

Gradient Ricci solitons are extensively studied and a few results on classifications of gradient Ricci soliton and some of them with warped product structures have been studied in [13-23]. Our aim of this paper to find the classification of derived inequality (1) attaining the equality cases by using the tool of gradient Ricci solitons, in terms of the squared norm of second fundamental form and constant sectional curvature. Consequently, we consider the following question: "What are necessary and sufficient conditions for warped product immersions in Kenmotsu space forms to be an Einstein warped product manifold with the impact of gradient Ricci soliton by using inequality (1)?" The answer, by assuming the equality case in the inequality (1) and a vector field $X$ is the gradient of the warping function of warped product manifold. We prove the following result which is one of the most important results in this direction.

Theorem 2. Let $\chi: M^{n}=N_{T}^{n_{1}} \times_{f} N_{\theta}^{n_{2}}$ be an isometric immersion of a warped product semi-slant submanifold $N_{T}^{n_{1}} \times_{f} N_{\theta}^{n_{2}}$ admitting gradient Ricci soliton into a Kenmotsu space form $\tilde{M}^{2 m+1}(c)$ with the following equality holds

$$
\begin{aligned}
\sum_{i=1}^{n_{1}} \sum_{j=1}^{n_{2}}\left\|h_{\mu}\left(e_{i}, e_{j}\right)\right\|^{2}+n_{1} n_{1} \lambda= & n_{2} \sum_{i=1}^{d_{1}}\left(\operatorname{Ric}\left(e_{i}, e_{i}\right)+\operatorname{Ric}\left(\varphi e_{i}, \varphi e_{i}\right)\right) \\
& +\left(\frac{c-3}{4}\right) n_{1} n_{2}-\left(\frac{c+1}{4}\right) n_{2}+1
\end{aligned}
$$

for any positive constant $\lambda>0$. Then one of the following conditions holds

(i) The warped product semi-slant submanifold $N_{T}^{n_{1}} \times_{f} N_{\theta}^{n_{2}}$ is an Einstein warped product of a Kenmotsu space form.

(ii) The slant angle of warped product semi-slant submanifold is satisfied $\theta=\cos ^{-1}\left(\sqrt{n_{2}}\right)$.

If $M^{n}$ admitting steady gradient Ricci soliton, we obtain such a classification theorem.

Theorem 3. Let $\chi: M^{n}=N_{T}^{n_{1}} \times_{f} N_{\theta}^{n_{2}}$ be an isometric immersion of a warped product semi-slant submanifold $N_{T}^{n_{1}} \times_{f} N_{\theta}^{n_{2}}$ admitting steady gradient Ricci soliton into a Kenmotsu space form $\widetilde{M}^{2 m+1}(c)$. If the following equality satisfies

$$
\begin{aligned}
\sum_{i=1}^{n_{1}} \sum_{j=1}^{n_{2}}\left\|h_{\mu}\left(e_{i}, e_{j}\right)\right\|^{2}= & n_{2} \sum_{i=1}^{d_{1}}\left(\operatorname{Ric}\left(e_{i}, e_{i}\right)+\operatorname{Ric}\left(\varphi e_{i}, \varphi e_{i}\right)\right) \\
& +\left(\frac{c-3}{4}\right) n_{1} n_{2}-\left(\frac{c+1}{4}\right) n_{2}+1
\end{aligned}
$$

Then the warped product semi-slant submanifold $N_{T}^{n_{1}} \times_{f} N_{\theta}^{n_{2}}$ is an Einstein warped product submanifold of Kenmotsu space forms or the slant angle of warped product semi-slant submanifold satisfies the condition $\theta=\cos ^{-1}\left(\sqrt{n_{2}}\right)$.

We shall study Ricci curvature on the structure of warped products. One fundamental question arises: What is the geometric meaning of Ricci curvature in Riemannian Geometry? Answer: Geometrically, Ricci flat means solving the Einstein field equation of Riemannian manifold with vanishing cosmological constant. In fact, the Ricci tensor is related to the matter content of the universe via Einstein's field equation in general relativity theory. It is a part of the curvature of space-time that classifies the degree to which matter will tend to converge or diverge in time. Therefore, Ricci curvature is more important than Riemannian curvature in physics. We shall find geometric obstructions of the Ricci curvature and Ricci tensor in the warped product manifolds ( for detail see $[17,21,24]$ and references therein). Our purpose is to study the physical significance of these problems in terms of 
warping functions, so that our study may find the useful applications of derived inequality in physics as we present the following result.

Theorem 4. Assume that $\chi: M^{n}=N_{T}^{n_{1}} \times_{f} N_{\theta}^{n_{2}}$ is an isometric immersion of a compact warped product semi-slant submanifold $N_{T}^{n_{1}} \times_{f} N_{\theta}^{n_{2}}$ into Kenmotsu space form $\widetilde{M}^{2 m+1}(c)$ with satisfying the following equality for warped product submanifold $M^{n}$

$$
\begin{aligned}
\int_{M} \mathcal{R} i c(\nabla \ln f, \bullet) \mathrm{dV}= & \left\{\left(\frac{c-3}{4}\right) n_{1}-\left(\frac{c+1}{4}\right)+\cot ^{2} \theta+\csc ^{2} \theta\right\} \\
& -\frac{1}{n_{2}} \sum_{i=1}^{n_{1}} \sum_{j=1}^{n_{2}}\left\|h_{\mu}\left(e_{i}, e_{j}^{*}\right)\right\|^{2} .
\end{aligned}
$$

Then at least one of the statement is true for $\mathrm{M}^{n}$,

(i) The warped product semi-slant $N_{T}^{n_{1}} \times_{f} N_{\theta}^{n_{2}}$ is a CR-warped product in a Kenmotsu space form $\widetilde{M}^{2 m+1}(c)$.

(ii) The non-trivial warped product semi-slant submanifold $N_{T}^{n_{1}} \times_{f} N_{\theta}^{n_{2}}$ into a Kenmotsu space form $\widetilde{M}^{2 m+1}(c)$ is a simply Riemannian product of $N_{T}^{n_{1}}$ and $N_{\theta}^{n_{2}}$.

As its application to the Euler-Lagrange equation, we prove the following interesting result.

Theorem 5. Assume that $\chi: M^{n}=N_{T}^{n_{1}} \times_{f} N_{\theta}^{n_{2}}$ be an isometric immersion of a compact warped product semi-slant into Kenmotsu space form $\widetilde{M}^{2 m+1}(c)$. If the warping function has solution of the Euler-Lagrange equation, then the necessary and sufficient condition of the warped product $N_{T}^{n_{1}} \times_{f} N_{\theta}^{n_{2}}$ is trivial such that

$$
\sum_{i=1}^{n_{1}} \sum_{j=1}^{n_{2}}\left\|h_{\mu}\left(e_{i}, e_{j}\right)\right\|^{2}=n_{2}\left\{\left(\frac{c-3}{4}\right) n_{1}-\left(\frac{c+1}{4}\right)+\cot ^{2} \theta+1\right\}
$$

Moreover, we shall also prove other results related to the above study.

\section{Preliminaries}

The classifications of $(2 m+1)$-dimensional almost contact metric manifold and Kenmotsu manifolds are widely defined in [1]. Moreover, a Kenmotsu manifold $\widetilde{M}^{2 m+1}$ is said to be a Kenmotsu space form $\widetilde{M}^{2 m+1}(c)$ with constant $\varphi$-sectional curvature $c$ if and only if the Riemannian curvature tensor $\widetilde{R}$ is given by (see [25])

$$
\begin{aligned}
& \widetilde{R}(X, Y, Z, W)=\frac{c-3}{4}(g(Y, Z) g(X, W)-g(X, Z) g(Y, W)) \\
&+\frac{c+1}{4}(\eta(X) \eta(Z) g(Y, W)+\eta(W) \eta(Y) g(X, Z)-\eta(Y) \eta(Z) g(X, W) \\
&-\eta(X) g(Y, Z) \eta(W)+g(\varphi Y, Z) g(\varphi X, W)-g(\varphi X, Z) g(\varphi Y, W) \\
&+2 g(X, \varphi Y) g(\varphi Z, W))
\end{aligned}
$$

for any $X, Y, Z, W \in \mathfrak{X}(T \widetilde{M})$, where the symbol $\mathfrak{X}(T \widetilde{M})$ to denote the Lie algebra of vector fields on a manifold $\widetilde{M}^{2 m+1}$. Assume that $M^{n}$ is a submanifold of an almost contact metric manifold $\widetilde{M}^{2 m+1}$ with induced metric $g$. If $\nabla$ and $\nabla^{\perp}$ are the induced connections on the tangent bundle $T M$ and the normal bundle $T^{\perp} M$ of $M^{n}$, respectively. Thus the Gauss and Weingarten formulas are given by

$$
\text { (i) } \widetilde{\nabla}_{U} V=\nabla_{U} V+h(U, V) \text {, (ii) } \widetilde{\nabla}_{U} N=-\mathcal{A}_{N} U+\nabla_{U}^{\perp} N \text {, }
$$


for each $U, V \in \mathfrak{X}(T M)$ and $N \in \mathfrak{X}\left(T^{\perp} M\right)$, where $h$ and $\mathcal{A}_{N}$ are the second fundamental form and the shape operator corresponding to the normal vector field $N$ for the immersion of $M^{n}$ into $\widetilde{M}^{2 m+1}$, respectively. They are related as $g(h(U, V), N)=g\left(\mathcal{A}_{N} U, V\right)$, where $g$ is Riemannian metric on $\widetilde{M}^{2 m+1}$ as well as the metric induced on $M$.

Now, let $\phi$ be a differential function defined on compact submanifold $M^{n}$ without boundary such that $\partial M^{n}=\varnothing$. Thus we have

$$
\int_{M^{n}} \Delta \phi \mathrm{d} V=0
$$

such that $\mathrm{d} V$ denotes the volume of $M^{n}$ [26]. In case, let $M^{n}$ be a compact Riemannian manifold with or without boundary. Then the kinetic energy function is defined in [27] as follows:

$$
E(\phi)=\frac{1}{2} \int_{M^{n}}\|\nabla \phi\|^{2} \mathrm{~d} V
$$

The Euler-Lagrange equation which corresponds to the above function is defined as:

$$
L=\frac{1}{2}\|\phi\|^{2}
$$

Theorem 6. [27] The Euler-Lagrange equation for the Lagrangian function (14)

$$
\Delta \phi=0
$$

We refer to [1] for some definitions related to submanifolds in Kenmotsu manifolds such as totally umbilical submanifolds, totally geodesic submanifolds, CR-submanifolds and slant submanifolds.

Cabrerizo et al. [28] proved a characterization for a slant submanifold in a contact metric manifold.

Lemma 1. [28] Let $M^{n}$ be a submanifold of an almost contact metric manifold $\widetilde{M}^{2 m+1}$. Then $M^{n}$ is slant if and only if there exists a constant $\lambda \in[0,1]$ such that

$$
P^{2}=-\delta(I+\eta \otimes \xi)
$$

Furthermore, in such a case, we have $\delta=\cos ^{2} \theta$.

Similarly, Cabrerizio, et al. [29] defined the semi-slant submanifolds such that

Definition 3. A submanifold $M^{n}$ of an almost contact metric manifold (Kenmotsu manifold) $\tilde{M}^{2 m+1}$, is said to be a semi-slant submanifold if there exists two orthogonal distributions $\mathcal{D}$ and $\mathcal{D}^{\theta}$ such that

(i) $\quad T M=\mathcal{D} \oplus \mathcal{D}^{\theta} \oplus\langle\xi\rangle$, where $\langle\xi(p)>$ is a 1-dimensional distribution spanned by $\xi(p)$.

(ii) $\mathcal{D}$ is invariant, i.e., $\varphi(\mathcal{D}) \subseteq \mathcal{D}$,

(iii) $\mathcal{D}^{\theta}$ is a slant distribution with slant angle $\theta$.

Assuming $n_{1}$ and $n_{2}$ denote the dimensions of $\mathcal{D}$ and $\mathcal{D}^{\theta}$ of semi-slant submanifold in a Kenmotsu manifold $\widetilde{M}^{2 m+1}$, respectively. Then the following remarks and definitions are useful to prove our main results.

Definition 4. $M^{n}$ is called invariant and slant with $n_{2}=0$ and $n_{1}=0$, respectively.

Definition 5. $M^{n}$ is defined as a contact CR-submanifold if the slant angle $\theta=\frac{\pi}{2}$.

Definition 6. A proper semi-slant submanifold $M^{n}$ is characterized by the slant angle $\theta \in\left(0, \frac{\pi}{2}\right)$. 
Remark 2. The normal bundle $T^{\perp} M$ for semi-slant submanifold can be decomposed as: $T^{\perp} M=F \mathcal{D}^{\theta} \oplus \mu$ where $\mu$ be an invariant subspace under $\varphi$ of normal bundle $T^{\perp} M$.

\section{Main Results for Warped Product Semi-Slant and Their Applications}

In this section, we will discuss some geometric applications in various physical terms such as Euler-Lagrange equation, Ricci curvature and divergence of Hessian. Throughout the study of this paper, we consider warped product semi-slant submanifold of type $N_{T}^{n_{1}} \times_{f} N_{\theta}^{n_{2}}$ of a Kenmotsu manifold, for classifications when structure vector $\xi$ is tangent to the base manifold $N_{T}^{n_{1}}$. In this direction we have the following important result;

Lemma 2. Let $\chi: M^{n}=N_{T}^{n_{1}} \times_{f} N_{\theta}^{n_{2}} \longrightarrow \widetilde{M}^{2 m+1}(c)$ be an isometric immersion of a proper warped product semi-slant submanifold $N_{T}^{n_{1}} \times_{f} N_{\theta}^{n_{2}}$ into a Kenmotsu space form $\widetilde{M}^{2 m+1}(c)$. If the equality is satisfied in the inequality (1), then we have

$$
\begin{aligned}
& \left(\frac{c-3}{4}\right) n_{1} n_{2}-\left(\frac{c+1}{4}\right) n_{2}+n_{2}+n_{2} \cot ^{2} \theta=\sum_{i=1}^{n_{1}} \sum_{j=1}^{n_{2}}\left\|h_{\mu}\left(e_{i}, e_{j}\right)\right\|^{2} \\
& +2 \cot ^{2} \theta\|\nabla \ln f\|^{2}+n_{2} \Delta(\ln f) \text {. } \\
& \frac{1}{n_{1}} \sum_{i=1}^{n_{2}} \sum_{j=1}^{n_{2}}\left\|h_{\mu}\left(e_{i}, e_{j}\right)\right\|^{2}=\sum_{i=1}^{\alpha}\left(\operatorname{Hess}(\ln f)\left(e_{i}, e_{i}\right)+\operatorname{Hess}(\ln f)\left(\varphi e_{i}, \varphi e_{i}\right)\right) \\
& +\frac{1}{n_{2}}+2\left(n_{2}-\cot ^{2} \theta\right)\|\nabla \ln f\|^{2} \\
& +\left(\frac{c-3}{4}\right) n_{1}-\left(\frac{c+1}{4}\right)+2 \cot ^{2} \theta+1 \text {. }
\end{aligned}
$$

Proof. The proof of the above lemma can seen in (see [1], Equation (6.4) and the proof Theorem 1.2) when the equality sign holds in the inequality (1).

\section{Applications of the Inequality (1) to Euler-Lagrange Equation}

Theorem 7. Assume that $\chi: M^{n}=N_{T}^{n_{1}} \times_{f} N_{\theta}^{n_{2}}$ is an isometric immersion of a warped product semi-slant into Kenmotsu space form $\widetilde{M}^{2 m+1}(c)$. If the warping function has the solution of Euler-Lagrange equation. Then, the necessary condition of $M^{n}$ to be a trivial warped product, i.e.,

$$
\|h\|^{2} \geq\left(\frac{c-3}{2}\right) n_{1} n_{2}-\left(\frac{c+1}{2}\right) n_{2} .
$$

Proof. Due to satisfying the condition of the Euler-Lagrange equation and from Theorem 6, we conclude that

$$
\Delta(\ln f)=0,
$$

Thus from (1) and (20), we derive

$$
\|h\|^{2} \geq\left(\frac{c-3}{2}\right) n_{1} n_{2}-\left(\frac{c+1}{4}\right) n_{2}+n_{2}\|\nabla \ln f\|^{2} .
$$

If the inequality (19) is satisfied, then (21) implies the warping function $\|\nabla \ln f\|^{2} \leq 0$. This means that $f$ must be constant on $M^{n}$. The proof is completed. 
Corollary 1. Let $\chi: M^{n}=N_{T}^{n_{1}} \times_{f} N_{\theta}^{n_{2}}$ is an isometric immersion of a warped product semi-slant into Kenmotsu space form $\widetilde{M}^{2 m+1}(c)$. If the warping function has the solution of Euler-Lagrange equation, then the necessary condition of $M^{n}$ to be a trivial warped product is

$$
\sum_{i=1}^{n_{1}} \sum_{j=1}^{n_{2}}\left\|h_{\mu}\left(e_{i}, e_{j}\right)\right\|^{2}=n_{2}\left\{\left(\frac{c-3}{4}\right) n_{1}-\left(\frac{c+1}{4}\right)+1+\cot ^{2} \theta\right\} .
$$

Proof. The proof follows from Theorem 6 and using (17) when equality holds in the inequality (1).

\subsection{Proof of the Theorem 5}

The proof of the above theorem follows the Theorem 7 by using (17) and Theorem 6 in case when the equality holds in inequality (1). This completes proof of the theorem.

\section{Applications to gradient Ricci soliton}

If $M^{n}$ is a gradient Ricci soliton warped product semi-slant submanifold of Kenmotsu space forms $\widehat{M}^{2 m+1}(c)$ with the potential function is a warping function $\ln f$, then we prove some classification theorems such as:

\subsection{Proof of the Theorem 2}

Assume that the warped product semi-slant submanifold $M^{n}$ of Kenmotsu space form $\widetilde{M}^{2 m+1}(c)$ satisfies fundamental Equations of the Ricci tensor or gradient Ricci soliton equation such that $\sigma=\ln f$

$$
\operatorname{Ric}=\lambda g+\operatorname{Hess}(\sigma),
$$

for any positive constant $\lambda>0$ and Hessian tensor $\operatorname{Hess}(\sigma)$ for the warping function $\ln f$. As we know that Ricci tensor and Hessian tensor are symmetric $(0,2)$ tensor fields, then for any vector fields $X$ and $Y$ are tangent to $N_{T}^{n_{1}}$, then (23) implies that

$$
\operatorname{Ric}(X, Y)=\lambda g(X, Y)+\operatorname{Hess}(\sigma)(X, Y)
$$

Assume that $\left\{e_{1}, \cdots e_{n_{1}}\right\}$ be an orthonormal frame for $N_{T}^{n_{1}}$ such that $\left\{e_{1}, e_{2}, \cdots e_{d_{1}}, e_{d_{1}+1}=\varphi e_{1}, \cdots e_{2 d_{1}}=\right.$ $\left.\varphi e_{d_{1}}, e_{2 d_{1}+1} \xi\right\}$. Let us substitute $X=Y=e_{i}$, for $1 \leq i \leq d_{1}$ in (24) and taking summation over the vector fields $N_{T}^{n_{1}}$, then it implies that

$$
\sum_{i=1}^{d_{1}} \operatorname{Ric}\left(e_{i}, e_{i}\right)=\lambda d_{1}+\sum_{i=1}^{d_{1}} \operatorname{Hess}(\sigma)\left(e_{i}, e_{i}\right)
$$

Replacing $e_{i}$ by $\varphi e_{i}$ in the above equation, we get

$$
\sum_{i=1}^{d_{1}} \operatorname{Ric}\left(\varphi e_{i}, \varphi e_{i}\right)=\lambda d_{1}+\sum_{i=1}^{d_{1}} \operatorname{Hess}(\sigma)\left(\varphi e_{i}, \varphi e_{i}\right)
$$

Thus from (25) and (26) it is easy to obtain that

$$
\sum_{i=1}^{d_{1}}\left(\operatorname{Ric}\left(e_{i}, e_{i}\right)+\operatorname{Ric}\left(\varphi e_{i}, \varphi e_{i}\right)\right)=2 d_{1} \lambda+\sum_{i=1}^{d_{1}}\left\{\operatorname{Hess}(\sigma)\left(e_{i}, e_{i}\right)+\operatorname{Hess}(\sigma)\left(\varphi e_{i}, \varphi e_{i}\right)\right\} .
$$


As the hypothesis of theorem, the equality case of the inequality (1) holds, we have the following equation from (18) of Lemma 2, i.e.,

$$
\begin{aligned}
\frac{1}{n_{2}} \sum_{i=1}^{n_{1}} \sum_{j=1}^{n_{2}}\left\|h_{\mu}\left(e_{i}, e_{j}\right)\right\|^{2}= & \sum_{i=1}^{d_{1}}\left[\operatorname{Hess}(\sigma)\left(e_{i}, e_{i}\right)+\operatorname{Hess}(\sigma)\left(\varphi e_{i}, \varphi e_{i}\right)\right] \\
& +2\left(n_{2}-\cot ^{2} \theta\right)\|\nabla \ln f\|^{2} \\
& +\left(\frac{c-3}{4}\right) n_{1} n_{2}-\left(\frac{c+1}{4}\right) n_{2}+1
\end{aligned}
$$

Follows to (27) and (28), we get

$$
\begin{aligned}
\sum_{i=1}^{n_{1}} \sum_{j=1}^{n_{2}}\left\|h_{\mu}\left(e_{i}, e_{j}\right)\right\|^{2}+n_{1} n_{1} \lambda= & n_{2} \sum_{i=1}^{d_{1}}\left(\operatorname{Ric}\left(e_{i}, e_{i}\right)+\operatorname{Ric}\left(\varphi e_{i}, \varphi e_{i}\right)\right) \\
& +2 n_{2}\left(n_{2}-\cot ^{2} \theta\right)\|\nabla \ln f\|^{2} \\
& +\left(\frac{c-3}{4}\right) n_{1} n_{2}-\left(\frac{c+1}{4}\right) n_{2}+1
\end{aligned}
$$

As the hypothesis $M^{n}$ is a warped product submanifold and satisfies the Equation (23), then Equation (29) provides two conditions such $\left(n_{2}-\cot ^{2} \theta\right)=0$, or $\|\nabla \ln f\|^{2}=0$. If we choose $\|\nabla \ln f\|^{2}=0$, then we get $\operatorname{grad}(\ln f)=0$, i.e., $f$ must be constant on $M^{n}$. Hence, by classification of gradient Ricci soliton with Remark $1, M^{n}$ is an Einstein warped product semi-slant submanifold in a Kenmotsu space form. On the other hand $\|\nabla \ln f\|^{2} \neq 0$, we get slant angle $\cot ^{2} \theta=\sqrt{n_{2}}$, which proves our assertion. It completes the proof of the theorem.

\subsection{Proof of the Theorem 3}

If we consider $\lambda=0$ in Equation (23) for steady gradient Ricci soliton, then proof follows the proof of Theorem 2.

\section{Applications to Ricci Curvature and Divergence of Hessian tensor}

In this section, we study some applications of derived inequality with equality cases and consider $M^{n}$ to be a compact Riemannian manifold with boundary.

\subsection{Proof of the Theorem 4}

Applying warping function $\sigma=\ln f$ to the well known Ricci identity from ([30], see page 159), we have

$$
\mathbf{D}^{2} d(\sigma)(Y, X, U)-\mathbf{D}^{2} d(\sigma)(X, Y, U)=d(\sigma) \mathbf{R}_{X Y} U,
$$

for every $X, Y, U \in \mathfrak{X}\left(T N_{T}\right)$. Since $f$ is a smooth function defined on $N_{T}^{n_{1}}$ and $\mathbf{D}_{X Y}^{2}=\mathbf{D}_{X} \mathbf{D}_{Y}-\mathbf{D}_{\mathbf{D}_{X} Y}$ is the second order covariant differentiable operator. Moreover, curvature tensor working as a derivative is defined by $\mathbf{R}_{X} Y=-\mathbf{D}_{X} \mathbf{D}_{Y}+\mathbf{D}_{Y} \mathbf{D}_{X}+\mathbf{D}_{[X, Y]}$. As $d \sigma$ is closed, then it can be easily proved that $\mathbf{D}^{2} d(\sigma)(Y, X, U)=\mathbf{D}^{2} d(\sigma)(X, Y, U)$, for any vector fields $X, Y$, and $U$ are tangent to $N_{T}^{n_{1}}$. Now we consider $\left\{e_{1}, e_{2}, \cdots e_{n_{1}}\right\}$ is a local orthonormal frame on $N_{T}^{n_{1}}$ and fixed the point $x \in N_{T}^{n_{1}}$ such that $\mathbf{D}_{e_{i}} e_{j}(t)=0$, for $1 \leq i, j \leq n_{1}$. If we choose $\mathbf{D}_{e_{i}} X(t)=0$, for any vector field $X$ and taking the trace with respect to $Y$ and $U$ in $\mathbf{D}^{2} d(\ln f)(Y, X, U)=\mathbf{D}^{2} d(\ln f)(X, Y, U)$. Thus using (30), we get

$$
\sum_{i=1}^{n_{1}}\left(\mathbf{D}^{2} d(\sigma)\right)\left(e_{i}, e_{j}, X\right)=-d(\Delta(\sigma))(X)+\mathcal{R} i c(\nabla \sigma, X) .
$$


Now we easily solved the left hand side of the above equation, we get

$$
\sum_{i=1}^{n_{1}}\left(\mathbf{D}^{2} d(\sigma)\right)\left(e_{i}, e_{j}, X\right)=\operatorname{div}(\operatorname{Hess}(\sigma))(X)
$$

Thus follows from (30) and (32), we derive

$$
\operatorname{div}(\operatorname{Hess}(\sigma))(X)+d(\Delta(\sigma))(X)=\operatorname{Ric}(\nabla \sigma, X),
$$

which implies that

$$
\operatorname{div}(\operatorname{Hess}(\sigma))+d(\Delta(\sigma))=\operatorname{Ric}(\nabla \sigma, \bullet)
$$

As hypothesis of the theorem, $M^{n}$ is to be compact warped product submanifold with boundary, then taking integration along the volume element $\mathrm{dM}$, we get

$$
\Delta(\sigma)+\int_{M} \operatorname{div}(\operatorname{Hess}(\sigma)) \mathrm{dM}=\int_{M} \operatorname{Ric}(\nabla \sigma, \bullet) \mathrm{dM} .
$$

Assuming that the equality holds in the inequality (1), then from Equation (18) of Lemma 2, we have

$$
\begin{aligned}
n_{2} \Delta(\sigma)+2 n_{2} \cot ^{2} \theta\|\nabla \sigma\|^{2}+\sum_{i=1}^{2 d_{1}} \sum_{j=1}^{2 d_{2}}\left\|h_{\mu}\left(e_{i}, e_{j}^{*}\right)\right\|^{2}= & \left(\frac{c-3}{4}\right) n_{1} n_{2}-\left(\frac{c+1}{4}\right) n_{2} \\
& +n_{2} \csc ^{2} \theta+n_{2} \cot ^{2} \theta
\end{aligned}
$$

It follows from (34) and (35), we find the following relation

$$
\begin{aligned}
n_{2} \int_{M} \operatorname{Ric}(\nabla \sigma, \bullet) \mathrm{dM}-n_{2} \int_{M} \operatorname{div}(\operatorname{Hess}(\sigma)) \mathrm{dM}+ & 2 n_{2} \cot ^{2} \theta\|\nabla \sigma\|^{2} \\
= & \left(\frac{c-3}{4}\right) n_{1} n_{2}-\left(\frac{c+1}{4}\right) n_{2}+n_{2} \csc ^{2} \theta \\
& +n_{2} \cot ^{2} \theta-\sum_{i=1}^{2 d_{1}} \sum_{j=1}^{2 d_{2}}\left\|h_{\mu}\left(e_{i}, e_{j}^{*}\right)\right\|^{2} .
\end{aligned}
$$

Using the Green theorem on a compact manifold $M^{n}$ and the given smooth function $f: M \rightarrow \mathbb{R}$, one has $\int_{M} \Delta f d V=0$. Using the results of K. Yano and M. Kon from (see [26]), immediately follows: $\Delta f=-\operatorname{div}(\nabla f)$ and from Green lemma $\int_{M} \operatorname{div}(X) d V=0$, for any arbitrary vector field $X$ on $M^{n}$. Thus, we get $\int_{M} \operatorname{div}(\operatorname{Hess}(\ln f)) d V=0$, Hess $^{\ln f}$ is Hessian tensor of warped function (or Laplacian of $\ln f$ ), then last equation implies that

$$
\begin{aligned}
\int_{M} \mathcal{R} i c(\nabla \sigma, \bullet) \mathrm{dM}+ & \frac{1}{n_{2}} \sum_{i=1}^{2 d_{1}} \sum_{j=1}^{2 d_{2}}\left\|h_{\mu}\left(e_{i}, e_{j}^{*}\right)\right\|^{2}+2 \cot ^{2} \theta\|\nabla \ln f\|^{2} \\
& =\left(\frac{c-3}{4}\right) n_{1}-\left(\frac{c+1}{4}\right)+\csc ^{2} \theta+\cot ^{2} \theta
\end{aligned}
$$

Let the equality (8) satisfy, then from (36), we get the following condition

$$
2 \cot ^{2} \theta\|\nabla \sigma\|^{2}=0
$$

Therefore, from the above equation, we find two cases such that $\cot ^{2} \theta=0$, or $\|\nabla \sigma\|^{2}=0$. If we consider $\cot ^{2} \theta=0 \Longrightarrow \frac{\cos ^{2} \theta}{\sin ^{2} \theta}=0$, which implies that $\cos \theta=0 \Longrightarrow \theta=\frac{\pi}{2}$. From the Definition 
5, we conclude that $M^{n}$ is a CR-warped product submanifold of Kenmotsu space form $\widetilde{M}^{2 m+1}(c)$. This completes the proof of (i) of the theorem.

If we assume that $\|\nabla \ln f\|^{2}=0$, which means that $\nabla \ln f=0$, i.e., grad $\ln f=0$. It implies that $f$ is a constant function on $M^{n}$. Hence, from the Definition 2, it concludes that $M^{n}$ is a trivial warped product semi-slant submanifold of Kenmotsu space form $\widetilde{M}^{2 m+1}(c)$. This is the second part (ii) of the theorem.

We prove another interesting theorem in terms of gradient scalar curvature as follows.

Theorem 8. Assume that $\chi: M^{n}=N_{T}^{n_{1}} \times_{f} N_{\theta}^{n_{2}}$ be an isometric immersion of a compact warped product semi-slant $N_{T}^{n_{1}} \times_{f} N_{\theta}^{n_{2}}$ into Kenmotsu space form $\widetilde{M}^{2 m+1}(c)$. Let the following equality be satisfied for warped product submanifold $M^{n}$

$$
\begin{aligned}
\frac{1}{n_{2}} \sum_{i=1}^{n_{1}} \sum_{j=1}^{n_{2}}\left\|h_{\mu}\left(e_{i}, e_{j}^{*}\right)\right\|^{2}+\frac{1}{2} \int_{M}(\nabla \mathcal{R}) \mathrm{dV}=\left\{\left(\frac{c-3}{4}\right) n_{1}-\left(\frac{c+1}{4}\right)\right. \\
\left.+\cot ^{2} \theta+\csc ^{2} \theta\right\} .
\end{aligned}
$$

Then at one of the following cases holds for $\mathrm{M}^{n}$,

(i) The warped product semi-slant $N_{T}^{n_{1}} \times_{f} N_{\theta}^{n_{2}}$ is a CR-warped product isometrically immersed into Kenmotsu space form $\widetilde{M}^{2 m+1}(c)$.

(ii) The non-trivial warped product semi-slant submanifold $N_{T}^{n_{1}} \times_{f} N_{\theta}^{n_{2}}$ into Kenmotsu space form $\widetilde{M}^{2 m+1}(c)$ is a simply Riemannian product of $N_{T}^{n_{1}}$ and $N_{\theta}^{n_{2}}$ or trivial warped product submanifold.

Proof. The following relation was proved in [10] for steady Ricci soliton and gradient Ricci tensor such that

$$
\nabla \mathcal{R}=2 \mathcal{R i c}(\nabla f, \bullet)
$$

Thus using Equation (38) in Theorem 4, we get the proof of the required theorem.

Author Contributions: Both authors contributed equally to this research. The research was carried out by both authors. The manuscript was prepared together and they both read and approved the final version.

Funding: The authors extended their appreciation to "the Deanship of Scientific Research at King Khalid University" for funding this work through research groups program under grant number R.G.P.1/65/40.

Acknowledgments: We are thankful to the learned referees whose suggestions improved the paper in its present form.

Conflicts of Interest: The authors declare no conflict of interest.

\section{References}

1. Ali, A.; Laurian-Ioan, P. Geometry of warped product immersion of Kenmotsu space forms and its applications to slant immersions. J. Geom. Phys. 2017, 114, 276-290. [CrossRef]

2. Ali, A.; Lee, J.W.; Alkhaldi, A.H. Geometric classification of warped product submanifolds of nearly Kaehler manifolds with a slant fiber. Int. J. Geom. Methods. Mod. Phys. 2018. [CrossRef]

3. Ali, A.; Laurian-Ioan, P. Geometric classification of warped products isometrically immersed in Sasakian space forms. Math. Nachr. 2018, 292, 234-251.

4. Ali, A.; Ozel, C. Geometry of warped product pointwise semi-slant submanifolds of cosymplectic manifolds and its applications. Int. J. Geom. Methods Mod. Phys. 2017, 14, 1750042. [CrossRef]

5. Atceken, M. Contact CR-warped product submanifolds in Kenmotsu space forms. Bull. Iran. Math. Soc. 2013, 39, 415-429.

6. Chen, B.Y. CR-warped products in complex projective space with compact holomorphic factor. Monatshefte für Mathematik 2004, 141, 177-186. [CrossRef] 
7. Hasegawa, I.; Mihai, I. Contact CR-warped product submanifolds in Sasakian manifolds. Geom. Dedicata 2003, 102, 143-150. [CrossRef]

8. Mihai, I. Contact CR-warped product submanifolds in Sasakian space forms. Geom. Dedicata 2004, 109, 165-173. [CrossRef]

9. Chen, B.Y. Geometry of warped product submanifolds: A survey. J. Adv. Math. Stud. 2013, 6, 1-43.

10. Hamilton, R.S. Three-manifolds with positive Ricci curvature. J. Differ. Geom. 1982, 17, 255-306. [CrossRef]

11. Blaga, A.M.; Crasmareanu, M.; Ida, C. Poisson and Hamiltonian structures on complex analytic foliated manifolds. J. Geom. Phys. 2014, 78, 19-28. [CrossRef]

12. Bajan, C.L.; Crasmareanu, M. Second order parallel tensors and Ricci solitons in 3-dimensional normal paracontact geometry. Ann. Glob. Anal. Geom. 2014, 46, 117-127. [CrossRef]

13. Barros, A.; Ribeiro, E., Jr. Integral formulae on quasi-Einstein manifolds and applications. Glasg. Math. J. 2012, 54, 213-223. [CrossRef]

14. Borros, A.; Gomes, J.N. A compact gradient generalized quasi-Einstein metric with constant scalar curvature. J. Math. Anal. Appl. 2013, 401, 702-705. [CrossRef]

15. Barros, A.; Batista, R.; Ribeiro, E., Jr. Compact almost Ricci solitons with constant scalar curvature are gradient. Monatshefte für Mathematik 2014, 174, 29-39. [CrossRef]

16. Crasmareanu, M.; Laurian-Ioan, P. Ricci solitons on CR-submanifolds of maximal CR-dimension in complex projective space. Carpathiam J. Math. 2016, 32, 173-177.

17. Feitosa, F.E.S.; Freitas, A.A.; Gomes, J.N.V. On the construction of gradient Ricci soliton warped product. arXiv 2015, arXiv:1506.00342.

18. Ghose, A.; Sharma, R. Sasakian metric as a Ricci soliton and related results. J. Geom. Phys. 2014, 75, 1-6. [CrossRef]

19. Gomes, J.N.; Wang, Q.; Xiab, C. On the $h$-almost Ricci soliton. J. Geom. Phys. 2017, 114, 216-222.

20. Kim, J.R. Remarks on the warped product structure from the Hessian of a function. Mathematics 2018, 6, 275. [CrossRef]

21. Lemes de Sousa, M.; Pina, R. Gradient Ricci solitons with structure of warped product. Results Math. 2016, 71, 825-840. [CrossRef]

22. Ouakkas, S.; Djebbouri, D. Conformal maps, biharmonic maps, and the warped product Mathematics 2016, 4, 15. [CrossRef]

23. Torbaghan, S.M.K.; Rezali, M.M. F-harmonic maps between doubly warped product manifolds Mathematics 2017, 5, 20. [CrossRef]

24. Dobarro, F.; Unal, B. Curvature of multiply warped products. J. Geom. Phys. 2015, 55, 75-106. [CrossRef]

25. Arslan, K.; Ezentas, R.; Mihai, I.; Murathan, C. Contact CR-wraped product submanifolds in Kenmotsu space form. J. Korean Math. Soc. 2005, 42, 1101-1110. [CrossRef]

26. Yano, K.; Kon, M. CR-submanifolds of Kaehlerian and Sasakian Manifolds; Birkhauser: Boston, MA, USA, 1983.

27. Calin, O.; Chang, D.C. Geometric Mechanics on Riemannian Manifolds: Applications to Partial Differential Equations; Springer Science \& Business Media: Berlin, Germany, 2006.

28. Cabrerizo, J.L.; Carriazo, A.; Fernandez, L.M.; Fernandez, M. Slant submanifolds in Sasakian manifolds. Glasg. Math. J. 2000, 42, 125-138. [CrossRef]

29. Cabrerizio, J.L.; Carriazo, A.; Fernandez, L.M.; Fernandez, M. Semi-slant submanifolds of a Sasakian manifold. Geom. Dedicata 1999, 78, 183-199. [CrossRef]

30. Gallot, S.; Hulin, D.; Lafontaine, J. Riemannian Geometry; Springer: Berlin, Germany, 1987.

(c) 2019 by the authors. Licensee MDPI, Basel, Switzerland. This article is an open access article distributed under the terms and conditions of the Creative Commons Attribution (CC BY) license (http:/ / creativecommons.org/licenses/by/4.0/). 